\title{
Mono- and Multilayer Silicene-Type Honeycomb Lattices by Oriented Attachment of PbSe Nanocrystals: Synthesis, Structural Characterization, and Analysis of the Disorder
}

\author{
Joep L. Peters, ${ }^{\dagger}$ Thomas Altantzis, ${ }^{\ddagger}$ Ivan Lobato, ${ }^{\ddagger}$ Maryam Alimoradi Jazi, ${ }^{\dagger}$ Carlo van Overbeek, ${ }^{\dagger}$ (৫) \\ Sara Bals, ${ }^{\ddagger 0}$ Daniel Vanmaekelbergh, ${ }^{*}{ }^{\dagger}{ }^{\dagger}$ and Sophia Buhbut Sinai ${ }^{\dagger}$ \\ ${ }^{\dagger}$ Condensed Matter and Interfaces, Debye Institute for Nanomaterials Science, Utrecht University, P.O. Box 80000, 3508 TA \\ Utrecht, The Netherlands \\ ${ }^{\ddagger}$ EMAT, University of Antwerp, Groenenborgerlaan 171, B-2020 Antwerp, Belgium
}

Supporting Information

\begin{abstract}
Nanocrystal (NC) solids are commonly prepared from nonpolar organic NC suspensions. In many cases, the capping on the NC surface is preserved and forms a barrier between the NCs. More recently, superstructures with crystalline connections between the NCs, implying the removal of the capping, have been reported, too. Here, we present largescale uniform superstructures of attached PbSe NCs with a silicene-type honeycomb geometry, resulting from solvent evaporation under nearly reversible conditions. We also prepared multilayered silicene honeycomb structures by using larger amounts of PbSe NCs. We show that the twodimensional silicene superstructures can be seen as a crystallographic slice from a 3-D simple cubic structure. We describe the disorder in the silicene lattices in terms of the nanocrystals position and their atomic alignment. The silicene honeycomb sheets are large enough to be used in transistors and optoelectronic devices.
\end{abstract}

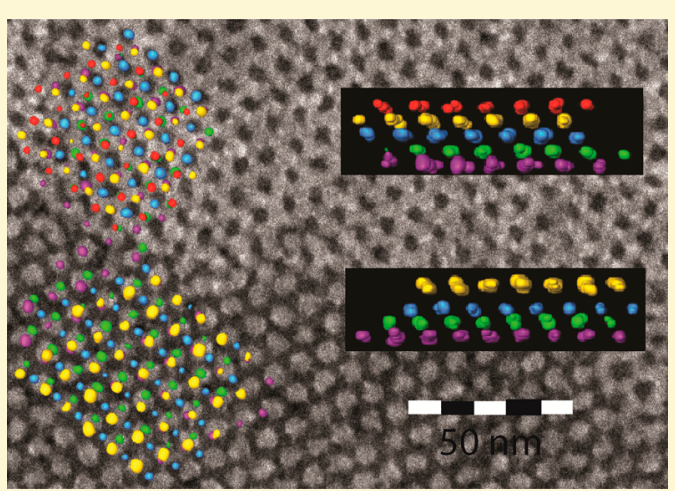

$\mathrm{T}$ hin solid films of semiconductor nanocrystals hold promise for several optoelectronic applications, such as LEDs, ${ }^{1,2}$ photodetectors, ${ }^{3}$ and solar cells. ${ }^{4-6}$ Commonly, nonpolar suspensions are used in which the nanocrystals are sterically stabilized by long organic capping molecules, and this capping is preserved in the NC solids. The resulting poor electronic coupling between the nanocrystals has been a major concern in the past years. Considerable progress has been made by smart surface chemistry, either on the level of the dispersed $\mathrm{NCs}^{7,8}$ or as a post-treatment on the nanocrystals present in the solid. ${ }^{9-11}$

In recent years, much effort has been put in the formation of nanocrystal solids in which the nanocrystals are epitaxially connected via specific facets. ${ }^{12-16}$ Since this attachment is crystallographic in nature, atomically coherent structures are fabricated with one (rods), ${ }^{12,17,18}$ two (sheets), ${ }^{12,13,16,19}$ or possibly three extended dimensions. ${ }^{20}$ Once the process of epitaxial attachment is under control, it might form a bottomup pathway for semiconductor structures, such as wires and quantum wells, complementary to the established but precious gas-phase methods. Moreover, it was shown recently that oriented attachment may result in two-dimensional structures with a superimposed square or honeycomb geometry. ${ }^{12}$ This is due to the specific truncated cubic shape of the PbSe nanocrystals and the precise attachment via (100) facets only. ${ }^{16}$ It has been theoretically predicted that the in-plane nanogeometry should strongly influence the semiconductor band structure. $^{21-24}$ The honeycomb structure for instance, with its similarity to graphene and silicene, results in Diractype valence and conduction bands with massless holes and electrons. ${ }^{23}$ To bring this scientific field forward and push it toward technological innovations, the chemical methodology of self-assembly and oriented attachment should be improved considerably for enhanced reproducibility, larger scale crystallographic domains, and full control of the nanogeometry.

Here, we show how to prepare silicene-type honeycomb structures from $\mathrm{PbSe}$ nanocrystals with lateral dimensions in the $10-100 \mu \mathrm{m}$ range in a reproducible manner. The process of NC self-assembly was changed such that it could occur under nearly reversible conditions. Solvent evaporation occurred under a gas atmosphere that was nearly saturated with the solvent, such that the entire process time slowed down to $16 \mathrm{~h}$. These nearly reversible conditions for the 2-D nanocrystal phases that precede the oriented $\mathrm{NC}$ attachment resulted in reproducible formation of large-scale 2-D silicene honeycomb domains. Moreover, by casting more $\mathrm{PbSe}$ nanocrystal building blocks on the liquid substrate, we can

Received: May 23, 2018

Revised: July 3, 2018

Published: July 3, 2018 
extend the silicene structures in the third (vertical) dimension. We performed high angle annular dark-field scanning transmission electron microscopy (HAADF-STEM) tomography experiments and acquired selected area electron diffraction (SAED) patterns along different tilt angles revealing the interrelation between all $\mathrm{PbSe} \mathrm{NC}$ structures observed so far: The two-dimensional silicene superstructure with three connections per NC and the planar square structures with four connections per NC can be considered as crystallographic slices taken from the 3-D simple cubic superlattice. Finally, we studied the disorder on the level of the nanocrystal positions in the silicene lattice and on the level of atomic plane misalignment due to imperfect epitaxy.

\section{METHODOLOGY}

There are two main methods to induce oriented attachment between $\mathrm{Pb}$-chalcogenide NCs. The first method uses amines to L-type displace the Z-type $\mathrm{Pb}$ (oleate) $)_{2}$ ligand and thereby induce attachment between (100) facets of the NCs. ${ }^{13,14,20,25}$ In the second method, a Pb-chalcogenide $\mathrm{NC}$ suspension in a nonpolar solvent is casted on ethylene glycol [EG], functioning as a liquid substrate. Solvent evaporation results in the formation of 2-D sheets that consist of domains with crystals attached in a linear fashion, a square geometry, and a honeycomb geometry. ${ }^{12}$

Our group has used the second method and has put the reaction system directly under a nitrogen atmosphere in a glovebox, resulting in relatively fast solvent evaporation and formation of a solid superstructure (typical process times were 20-40 min). We obtained sheets of a NC monolayer thickness in which the NCs were epitaxially attached; the sheets showed domains with a square and/or honeycomb geometry. The method of fast evaporation has, however, not been optimized and lacks the reproducibility required for technological innovation: The relative amounts of square and honeycomb structures varied considerably with the experiment, and the size of the superstructure domains was rather small $(<1 \mu \mathrm{m})$ and also varied from experiment to experiment.

Here, we show how we optimized the synthesis of honeycomb superstructures by choosing for very slow process conditions, i.e., very slow evaporation of the toluene solvent from the nanocrystal dispersion (see Figure 1), which is under a gas atmosphere nearly saturated with toluene. A Petri dish is fully filled with EG, creating a flat liquid surface. This Petri dish is then placed in a second larger Petri dish filled with $2 \mathrm{~mL}$ of toluene. A PbSe NC dispersion in toluene with a known concentration and size determined by FT-IR $(5-6.3 \mathrm{~nm}$ in size) is drop-casted on top of the EG liquid substrate, and both Petri dishes are covered with a beaker. This entire handling is performed in a glovebox with $<0.1 \mathrm{ppm}$ oxygen. Because of the presence of a toluene liquid phase in this setup, the gas atmosphere above the NC dispersion has a constant and large toluene partial pressure; this slows down the process of solvent evaporation enormously. We collected the NC sheets typically after $16 \mathrm{~h}$ of process time.

\section{TWO-DIMENSIONAL SILICENE SHEETS AS THE DOMINANT PHASE}

Figure 2 shows a typical picture of the samples obtained with the method explained above when the amount of NCs dropcasted was chosen to form a monolayer system. The optical microscope image shows large dark blue colored domains

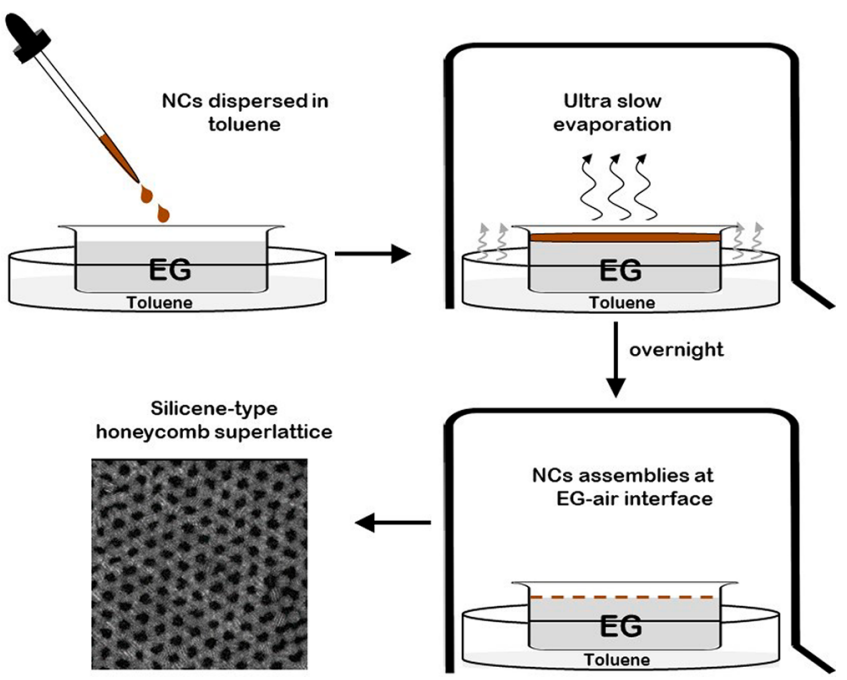

Figure 1. Schematic representation of the experimental setup and methodology. In the first step a $6.5 \mathrm{~mL}$ Petri dish is filled with ethylene glycol serving as a liquid substrate. This dish is then placed in a larger Petri dish containing $2 \mathrm{~mL}$ of toluene. A PbSe $\mathrm{NC}$ suspension with a controlled amount of NCs is drop-casted on the EG substrate, and a beaker is placed on top to slow down the evaporation. Solvent evaporation and superlattice formation take $16 \mathrm{~h}$. The NC sheet that is formed on the liquid substrate is transferred onto a desire substrate or TEM grid. The entire handling is performed in a glovebox with less than $0.1 \mathrm{ppm}$ oxygen.

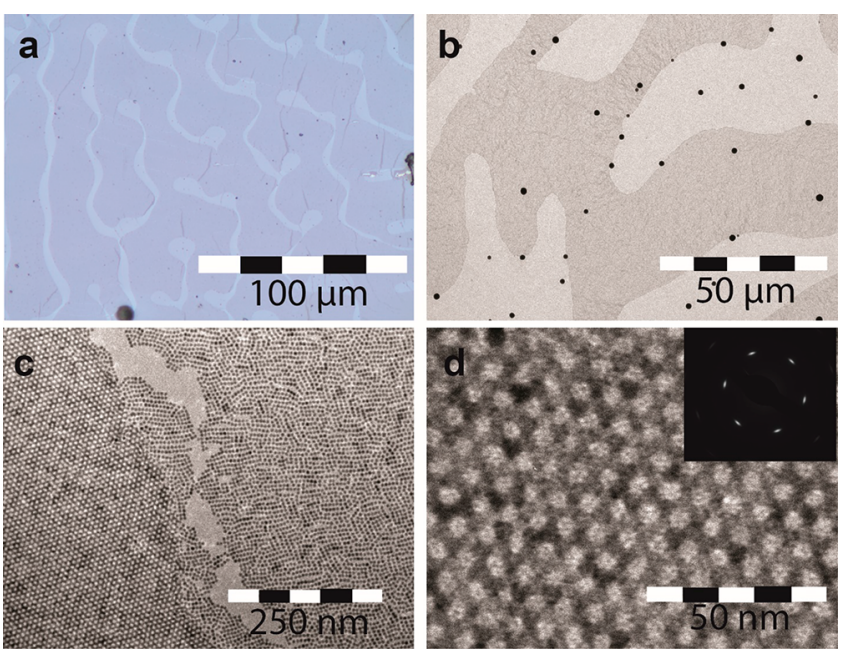

Figure 2. Global and zoomed-in images of the PbSe sheets formed by slow processing. (a) Optical microscope image showing large uniform domains $(>10 \mu \mathrm{m}$, dark blue) surrounded by thinner sheets (pale blue). (b) Low-magnification TEM image showing similar uniform domains (dark gray) surrounded by domains with a lower density of nanocrystals. (c) A zoomed-in TEM image showing that the denser domains have a honeycomb periodicity, while the paler regions consist of square or linear structures with a thickness of a nanocrystal monolayer. (d) High-magnification TEM image of the honeycomb structure; the inset shows a SAED pattern with six spots on the 220 ring, proving that the nanocrystals are aligned with their $\langle 111\rangle$ crystallographic axis upward and also aligned in plane.

surrounded by a paler background. The dark-blue domains have dimensions in the $10-100 \mu \mathrm{m}$ range and boundaries with a nearly minimized contact line (see Figure S1 for more examples). Here, solvent evaporation must be slower than the diffusion of NCs in a lateral plane, and nanocrystals can find 
positions of minimal energy. ${ }^{26-28}$ It is reasonable to assume that all processes that precede oriented attachment, i.e., the formation of 2-D NC structures with a specific geometry and nanocrystal orientation induced by desorption of the capping ligands from the (100) facets, have time to occur under natural reaction conditions, i.e., without transport limitations. We remark that faster solvent evaporation results in irregular drying patterns, coffee stains, ${ }^{29-34}$ or even fractal structures. $^{28,35}$ For convenience, we have repeated the experiments under fast solvent evaporation and observed coffee stain structures and fractal domains consisting of linear, square, and honeycomb superstructures (Figures S2 and S3).

By a more detailed inspection of the transmission electron microscopy (TEM) images in Figure 2 it becomes clear that the darker structures have a honeycomb geometry, while the lighter regions consist of a monolayer film of $\mathrm{PbSe} \mathrm{NCs}$ attached in linear and square structures. The darker blue regions in the optical images must correspond to the darker regions on the TEM images and indicate a higher NC density. The proportion of darker area varies between 30 and $70 \%$, when examined for repetitions of the same experiment, and sometimes such variations were even present on the examined regions of a single TEM grid. We remark here that the dark blue regions can contain several honeycomb domains. The Fourier transforms of Figure S7 show that they can extend over several micrometers. The grain boundaries between the domains are discussed below. The black spots present in Figure $2 \mathrm{~b}$ are analyzed with EDX and appear to be selenium rich (Figure S4). Electron diffraction shows that in the honeycomb domains all the nanocrystals are aligned with the $\langle 111\rangle$ direction perpendicular to the plane and that the (100) planes are also aligned. The observation of attached NCs in the linear structures (right side of Figure 2c) and aligned NC via SAED indicates that the PbSe NCs in these honeycomb structures are epitaxially connected. Since the TEM images in Figure 2 only correspond to $2 \mathrm{D}$ projections, electron tomography was applied to obtain conclusive evidence in $3 \mathrm{D}$ (see Figure $3 \mathrm{~d}$ ). ${ }^{36}$ Similar honeycomb structures have been previously reported and identified to have a buckled silicenelike geometry, $2 \mathrm{NC}$ monolayers (2 ML) in thickness. ${ }^{16} \mathrm{We}$ can conclude that slow solvent evaporation results in a substantial improvement with respect to the previous fast procedure, both regarding the reproducibility and specificity of the final product (i.e., the desired honeycomb lattice) and the lateral dimensions of the domains, now in the $100 \mu \mathrm{m}$ range instead of $0.1-5 \mu \mathrm{m}$.

\section{MULTILAYER SILICENE STRUCTURES}

When we increased the amount of NCs casted on the EG, solid films thicker than 1-2 NC ML should necessarily be formed. The question is then if the nanocrystals are also epitaxially connected, and what type of NC solids form? A typical result is presented and analyzed in Figure 3. Figure 3a shows a multilayer structure with a sequence of terraces, very similar to what can be found on the surface of atomic solids. The multilayer domains can be tens of micrometers in the lateral dimensions and up to 10 layers in the vertical dimension. Such domains are surrounded by sheets of buckled silicene-like structures. SAED acquired from each of the terraces shows that the NCs are similarly oriented, indicating that the entire superstructure with all its terraces is in fact a single crystal with buckled silicene geometry. This is corroborated by the HAADF-STEM image of Figure $3 c$ which shows the step
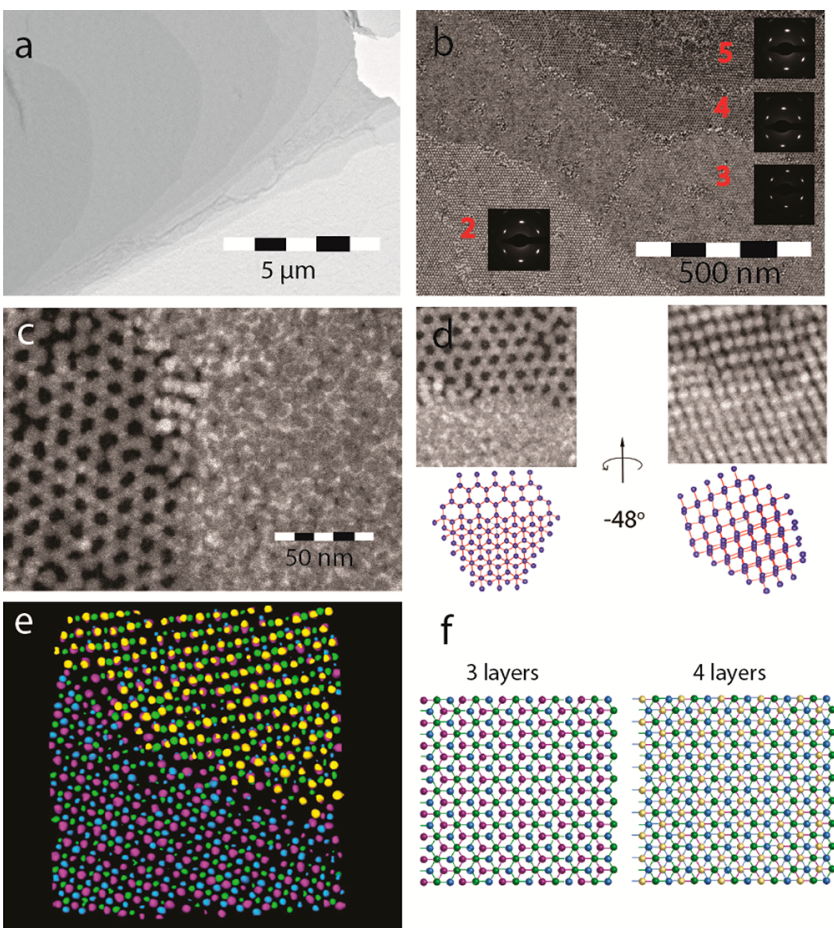

f

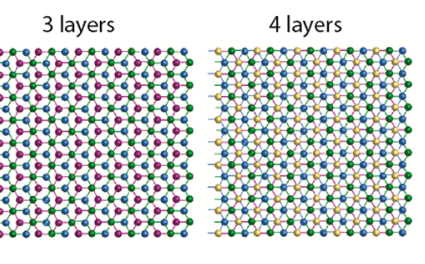

Figure 3. Structural analysis of multilayered superstructures formed under nearly reversible conditions. (a) TEM image showing a multilayered domain; terraces indicate the number of NCs in the vertical direction. (b) TEM image of thinnest border region (2-5 ML) show honeycomb type structures with all NCs oriented with the $\langle 111\rangle$ axis upward, as indicated by the SAED. (c) HAAFD-STEM image of a step region between a 2 and $3 \mathrm{ML}$ silicene structure. (d) Tilting of the sample over $48^{\circ}$ shows the crystallographic necks between NCs and bond angles of $90^{\circ}$. (e) A 3D visualization of the reconstructed volume of a region between 3 and $4 \mathrm{ML}$, with the NCs of the bottom, second, third, and fourth layers indicated by purple, green, blue, and yellow colors, respectively. (f) The $3 \mathrm{ML}$ structure constitutes an $\mathrm{ABC}$ stacking, whereas the $4 \mathrm{ML}$ structure an $\mathrm{ABCA}$ stacking, in agreement with the coherent multilayered silicene geometry.

between a visible silicene honeycomb (the $2 \mathrm{ML}$ layer structure) and a $3 \mathrm{ML}$ structure. By changing the angle of the sample with respect to the electron beam, it is shown that the nanocrystals are connected via epitaxial necks, in the $2 \mathrm{ML}$ structure and in the $3 \mathrm{ML}$ superstructure as well. This is also shown for the region between the 3 and $4 \mathrm{ML}$ in Figure S5. Electron tomography series were acquired of this specific region, and a $3 \mathrm{D}$ visualization of the manually segmented reconstruction is presented in Figure 3e. From the reconstruction, the number of layers can be determined as well as their $3 \mathrm{D}$ stacking along the axis perpendicular to the substrate. Moreover, a grain boundary was observed between a domain with three layers and one with four layers of NCs. Furthermore, each of the bottom NCs (purple) is surrounded in a trigonal way by three NCs of the second layer. Similarly, the NCs of the second layer (green) are surrounded by three NCs of the third layer (blue), meaning that an $\mathrm{ABC}$ order is present in the vertical direction. In the other grain, NCs of the fourth layer are above those of the first layer, i.e., an ABCA order. This is in complete agreement with a multilayered superstructure with silicene-type geometry. The idealized schemes in Figure $3 \mathrm{f}$ show the structures consisting of three and four layers of NCs. The same analysis is also performed for 
the transition region between two domains with four and five layers of NCs, shown in Figure S6. For the structure that consists of five layers of NCs we observe an overall $A B C A B$ stacking. This reveals that our multilayered silicene structures are, in fact, a part of a simple cubic NC superstructure. This also means that the buckled $2 \mathrm{ML}$ silicene honeycomb structure can be considered as a crystallographic slice taken from a simple cubic structure. ${ }^{37}$ In the $2 \mathrm{ML}$ silicene structure, the NCs have three neighbors connected via $\mathrm{NC}(100)$ / (100)NC epitaxy, with bonding angles of $90^{\circ}$. We would like to remark here that atomic crystals with simple cubic structure are extremely rare limited to special elements, such as polonium. While the atomic volume fraction of such a Po crystal is low (52\%), it is even lower in the NC superstructure $(\sim 32 \%)$ due to the necks between the NCs. ${ }^{16,38}$

\section{DEFECTS IN THE HONEYCOMB STRUCTURE, CONSIDERING THE NANOCRYSTALS AS ATOMIC SITES}

Although the superstructures appear regular and periodic over a very large number of unit cells as shown by Fourier transform analysis (Figure S7), several types of disorder can be observed. Here, we first make abstraction of the atomic structure of the nanocrystals; i.e., we consider the nanocrystals as "artificial atom sites" of a silicene structure. The most obvious "artificial atom" defects that we observe are presented in Figure 4. Figure
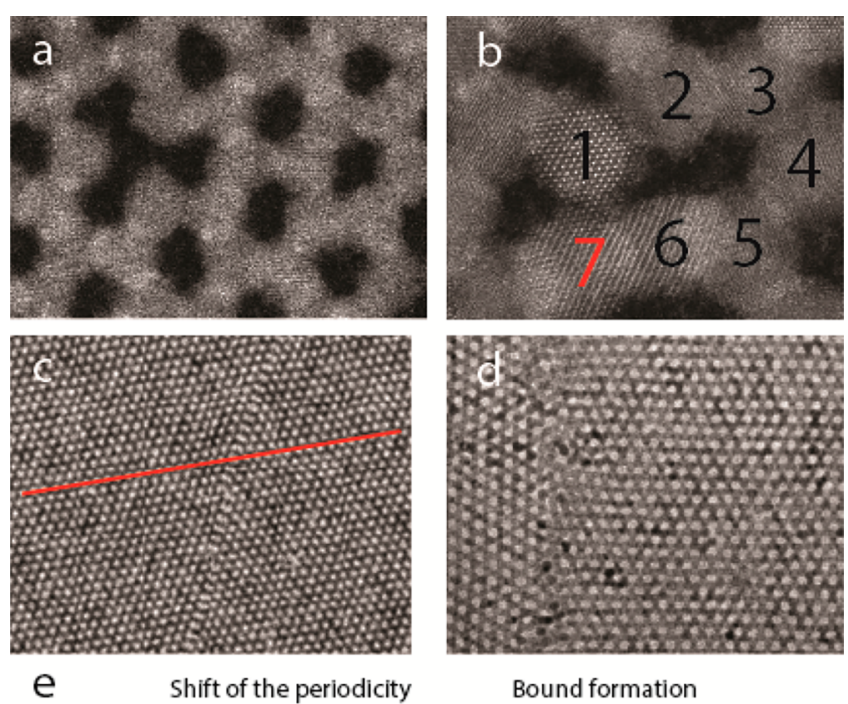

Bound formation
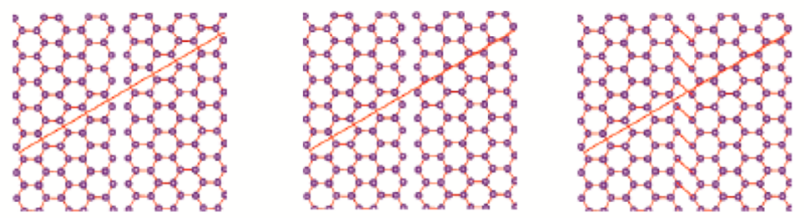

Figure 4. Overview of several types of "artificial atom (= nanocrystal)" defects in a two monolayer silicene lattice. (a) A missing NC in the silicene structure, i.e., vacancy. (b) An NC interstitial causes a strained 7-ring in the silicene structure. In (c) a one-dimensional defect is observed and modeled in (e); the defect finds its origin in a shift of the right silicene lattice over half a unit cell with respect to the left lattice, while both lattices retain the same crystallographic orientation (see cartoon). A ball and stick model is shown in the Supporting Information, Figure S9. (d) A disordered grain boundary region between two silicene lattices that have a different crystallographic orientation. 4a shows a missing NC in the silicene structure, i.e., a vacancy. Figure $4 \mathrm{~b}$ shows a ring with seven NCs (instead of the regular six) probably due to incorporation of an extra NC in the silicene structure. We should remark here that similar point defects have been observed in graphene ${ }^{39}$ and NC assemblies. $^{40}$ Figure $4 \mathrm{c}$ shows a remarkable one-dimensional line defect that connect silicene lattices with the same crystallographic orientation, which are however shifted with respect to each other. Another example of this defect is shown in Figure S8, where a part of the honeycomb lattice is shifted causing a similar line defect. The fact that the bonding configuration is possible with only NC (100)/(100)NC epitaxy, though with strain, is also proved with a ball-andstick chemistry model (Figure S9). Also, more classic grain boundaries can be observed that connect silicene domains with a different orientation as presented in Figure $4 \mathrm{~d}$.

\section{MORE COMPLEX DEFECTS}

Besides the zero- and one-dimensional defects presented above, also more complex defects appear in the honeycomb structure with the double square and zigzag structures ${ }^{41}$ as prominent examples (Figure S10a,b). Those structures often form a boundary between two silicene domains but can also be present within one honeycomb grain. In the latter case, we could spot regions in which the honeycomb structure deviates from the hexagonal symmetry. The compressed regions with deviating geometry might be explained by NC monolayer movements along a specific direction (see Figures S8 and $\mathrm{S} 10 \mathrm{c}-\mathrm{f})$ prior to oriented attachment; ${ }^{42-45}$ a tentative model example is presented in Figure S11. The reason for those relatively collective $\mathrm{NC}$ motions could lie in local fluctuations in the solvent evaporation and suspension/air surface tension, agglomerations of capping ligands or impurities, or be the effect of sudden motions of the sample (a few tens of nanometers amplitude could be sufficient).

\section{DISORDER DUE TO NANOCRYSTAL MISALIGNMENT IN THE SILICENE LATTICE}

We now consider each of the nanocrystal sites as an ordered array of atoms. Perfect crystallographic epitaxy should lead to an exact orientation of the nanocrystals with the [111] axis perpendicular to the plane and also completely aligned in plane. There are, however, many occasions whereby $\mathrm{NC} / \mathrm{NC}$ attachment was not perfect, leading to local deviations in the alignment of the atomic lattices. ${ }^{46,47}$ This can be seen from high-resolution images (Figure 5a) and can also be deduced by the contrast difference between the attached NCs within the honeycomb lattice (Figure 2c,d). To study this type of disorder, we acquired selected area electron diffraction (SAED) patterns along different tilt angles. By measuring the width of the diffraction peaks, we obtained the average angular disorder in the orientation of the NC with respect to each other. An example is shown in Figure 5b, where on the left a typical SAED image of a honeycomb superstructure is shown, with six diffraction spots on the 220 ring. Azimuthal traces are made of these six spots and plotted in Figure $5 \mathrm{c}$ in blue. These are subsequently fitted with Gaussians in red and the width of the peaks is used as a measure of the angular disorder of the NCs. This width gives the angular variation of the rotation of the $\langle 111\rangle \mathrm{NC}$ axis with respect to the direction perpendicular to the plane. 

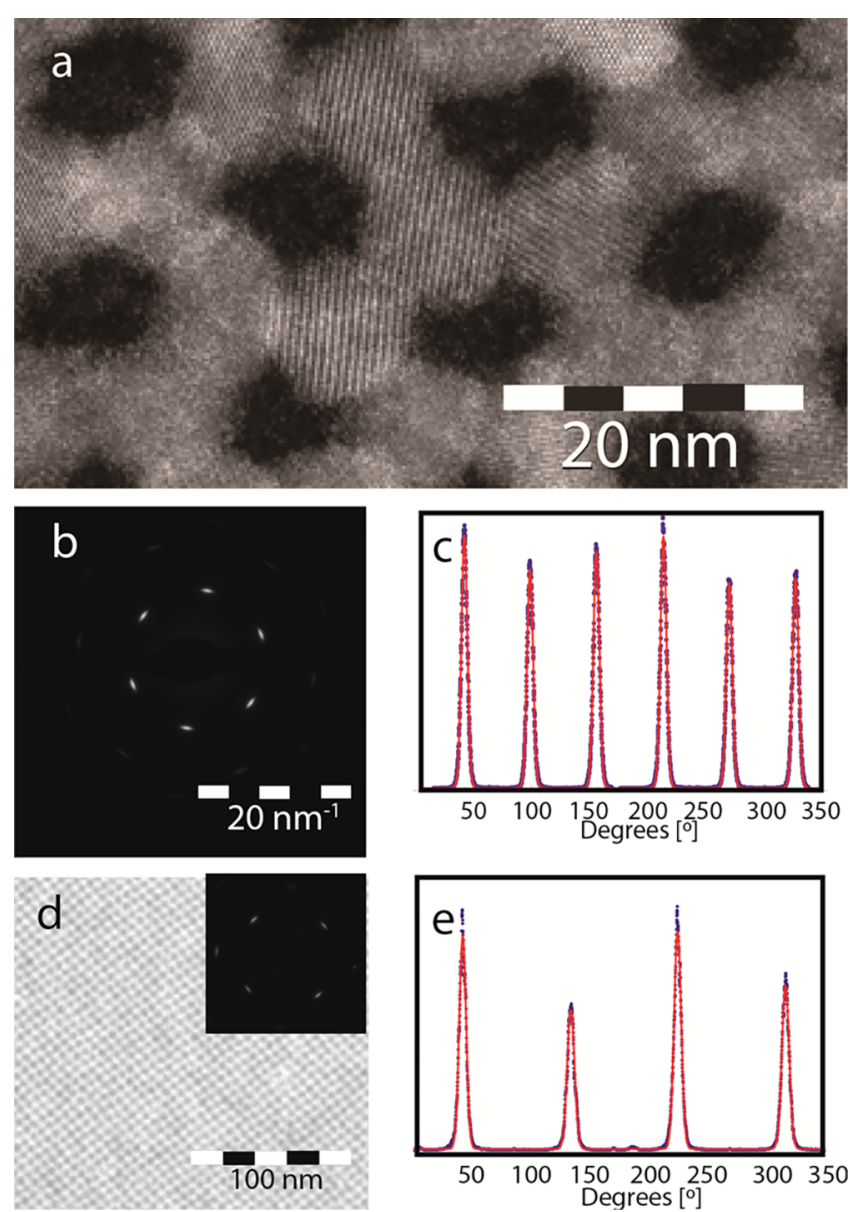

Figure 5. Analysis of NC misalignment in the silicene honeycomb lattice with SAED under two different angles. In (a) a HAADF-STEM image of a small fraction of the silicene honeycomb lattice is presented. The nonuniform crystallographic appearance indicates imperfect alignment of the NCs. (b) Typical SAED image with 6 spots on the 220 ring. (c) Plot of the spots as azimuthal traces fitted with Gaussians. The width of the Gaussians quantifies the degree of misalignment of the NCs with respect to the ideal silicene lattice. Parts $\mathrm{d}$ and e present a similar analysis after rotation of the sample with respect to the electron beam over $45^{\circ}$.

The full width at half-maximum of the plotted Gaussians is $3.1^{\circ}$. This means that $95 \%$ of the angles are within a $12.5^{\circ}(\sigma=$ $0.4)$ deviation from the ideal $\langle 110\rangle$ direction $((111)$ facet pointing upward). To test the reliability of the analysis, we measured the peak widths for a monocrystalline molybdenum crystal: $1.55^{\circ}$ for a $95 \%$ interval $\left(\sigma=0.44^{\circ}\right)$, averaged over three different locations (Figure S12). This width probably originates from a combination of instrument limitations and slight deviations from a perfect crystallographic orientation. The broadening is much smaller compared to that of the honeycomb superlattice, which indicates that broadening of the SAED peaks is a trustful representation of the averaged orientation disorder of the NCs in the superlattice.

The analysis of the angle distribution was also performed for a $45^{\circ}$ tilted sample orientation (see Figure S13) and is presented in Figure $5 \mathrm{~d}-\mathrm{f}$. In this way, the deviation of the $\langle 100\rangle$ axis from that in the ideal silicene structure can be obtained, yielding an angular variation of $15.3^{\circ}(\sigma=0.73)$. This result is in line with theoretical and experimental studies showing that the alignment of crystals is energetically favorable within a $15^{\circ}$ angle. $^{48,49}$ Notice that the $\langle 100\rangle$ directions constitute the directions of $\mathrm{NC}(100) /(100) \mathrm{NC}$ epitaxy and are all slightly out of plane. ${ }^{16}$ The values of the angular distribution along the $\langle 100\rangle$ direction are larger compared to those around the ideal $\langle 111\rangle$ direction. This is expected since the attachment process is the source of the disorder. The distribution in the $\langle 111\rangle$ direction results from positive and negative errors in the misalignment along the $\langle 100\rangle$ directions, yielding a lower value.

We performed the same measurements for the vertical extended $(3,4, \ldots$ NC ML) silicene lattices. The angular variation around the $\langle 110\rangle$ direction is $8.8^{\circ}(\sigma=0.14)$ and $12.6^{\circ}(\sigma=0.6)$ along the $\langle 100\rangle$ direction. Multilayer silicene superlattices thus show smaller deviations in the NC orientation, compared to the $2 \mathrm{ML}$ silicene lattice. Such a decrease of the disorder with increasing number of attached layers of NCs was also observed for square lattices and cubic lattices. $^{50}$

\section{SUMMARY}

We have shown that extremely slow solvent evaporation results in improved reproducibility and specificity regarding the formation of silicene honeycomb superlattices from PbSe NC suspensions. It is also possible to prepare multilayered silicene lattices. Using HAADF-STEM tomography, we show that the bilayer and multilayered silicene structures of attached $\mathrm{PbSe}$ nanocrystals form slices of the simple cubic superstructure. Our superstructures show a long-range honeycomb periodicity, but with several types of site defects. In principle, the superstructure domains should also be atomically coherent, but we show that atomic coherency is broken by misorientations due to nonideal epitaxial connections between the nanocrystals.

\section{METHODS}

Chemicals Used. Lead acetate trihydrate ( $\geq 99.99 \%$, trace metals basis), 1-octadecene (technical grade, 90\%), oleic acid (technical grade, $90 \%$ ), selenium powder (100 mesh, $99.99 \%$ trace metals basis), diphenylphosphine (98\%), trioctylphosphine (technical grade, 90\%), tetrachloroethylene (anhydrous, $\geq 99 \%$ ), toluene anhydrous (99.8\%), methanol anhydrous (99.8\%), and butanol anhydrous (99.8\%) were all purchased from Sigma-Aldrich.

Nanocrystal Synthesis. PbSe NC was synthesized via a method established earlier. ${ }^{1}$ In brief, $4.77 \mathrm{~g}$ of $\mathrm{Pb}\left(\mathrm{C}_{2} \mathrm{H}_{3} \mathrm{O}_{2}\right)_{2} \cdot 3 \mathrm{H}_{2} \mathrm{O}, 10.35 \mathrm{~g}$ of oleic acid, and $39.75 \mathrm{~g}$ of octadecene (ODE) were mixed and heated $\left(120{ }^{\circ} \mathrm{C}\right)$ under vacuum for $5 \mathrm{~h}$ to remove water and acetate. The selenium precursor was made by dissolving $3.52 \mathrm{~g}$ of Se powder in $0.41 \mathrm{~g}$ of diphenylphosphine and $46.59 \mathrm{~g}$ of trioctylphosphine. The selenium precursor was inject into a heated solution of the lead precursor $\left(180^{\circ} \mathrm{C}\right)$ under vigorous stirring. After $70 \mathrm{~s}$, the reaction was quenched via injection of $30 \mathrm{~mL}$ of methanol/butanol mixture $(1: 2)$. The dispersion was centrifuged and subsequently dissolved in toluene. A concentrated solution of $\mathrm{PbSe}$ nanocrystals (approximately $1.2 \times 10^{-4} \mathrm{M}$ ) was washed with methanol in a volume ratio of $1: 2$. The resulting brownish solution was centrifuged until a clear white solution was visible with a black precipitation. The clear solution was decanted, and the black precipitation was dissolved in toluene. The washing procedure was repeated twice more.

Superlattice Synthesis. The honeycomb superlattice was synthesized as described in the main text. A Petri dish was completely filled with $6.5 \mathrm{~mL}$ of EG, creating a flat surface. This Petri dish was placed in another bigger petridish containing $2 \mathrm{~mL}$ of toluene. Subsequently, $350 \mu \mathrm{L}$ of a diluted NCs solution $\left(7.7 \times 10^{-8} \mathrm{M}\right)$ in toluene was drop-casted on top of $6.5 \mathrm{~mL}$ of EG, and both Petri dishes were covered with a bigger beaker $(400 \mathrm{~mL})$. The honeycomb structure was formed on top of the EG in $16 \mathrm{~h}$ and could be transferred to any substrate. It is important that no high boiling point organics are 
present in the NC dispersion, since that will disturb the synthesis. The whole experiment is performed in a glovebox containing $<0.1 \mathrm{ppm}$ of $\mathrm{O}_{2}$ and free of amines or other volatile gases. The synthesis was successful for NCs with sizes in between 5 and $6.3 \mathrm{~nm}$.

Synthesis of the Multilayered Silicene Lattices. A Petri dish was completely filled with $6.5 \mathrm{~mL}$ of EG, creating a flat surface. This Petri dish was placed in another bigger Petri dish containing $6 \mathrm{~mL}$ of toluene. Subsequently, $700 \mu \mathrm{L}$ of a diluted NCs solution $\left(1.6 \times 10^{-7}\right.$ $\mathrm{M})$ in toluene was drop-casted on top of $6.5 \mathrm{~mL}$ of EG, and both Petri dishes were covered with a bigger beaker $(400 \mathrm{~mL})$. The honeycomb structure was formed on top of the EG in $64 \mathrm{~h}$ and could be transferred to any substrate. The other conditions are similar as in the honeycomb case described above.

FT-IR measurements were performed in a Bruker Vertex 70. A special airtight liquid cell (International Crystal Laboratories) was used with a path length of $0.5 \mathrm{~mm}$ and two $\mathrm{KBr}$ crystals at the back and front side to make it transparent for IR light. Spectra were recorded from 400 to $7500 \mathrm{~cm}^{-1}$, with a $\mathrm{KBr}$ beam splitter, a DLaTGS D301 detector, and a mid-IR source. For all measurements, tetrachloroethylene (TCE) was used as solvent.

SAED measurements were performed at a Philips Tecnai operating at $200 \mathrm{kV}$. It is essential for the reproducibility of the tilting series that the SAED peaks are approximately similar in size (see example in the main text).

HAADF-STEM images and electron tomography series were acquired on an aberration corrected "cubed" FEI Titan electron microscope operated at $300 \mathrm{kV}$. Electron tomography series were acquired manually within a tilt range from $-76^{\circ}$ to $+76^{\circ}$ and an increment of $2^{\circ}$, using a Fischione model 2020 single-tilt tomography holder. The reconstruction was performed using the simultaneous iterative reconstruction technique (SIRT) implemented in the ASTRA toolbox. ${ }^{51}$ The reconstructed series were segmented manually by using the AMIRA software.

\section{ASSOCIATED CONTENT}

\section{S Supporting Information}

The Supporting Information is available free of charge on the ACS Publications website at DOI: 10.1021/acs.chemmater.8b02178.

Optical and TEM images of the ultrathin PbSe films, TEM images of the PbSe NC films formed by fast toluene evaporation, fractal patterns, EDX analysis of the black spots, multilayered silicene honeycomb structures, tomography between the 4th and 5th layer, Fourier transform of the honeycomb periodicity, various types of grain boundaries, ball and stick model, examples of complex defects, lattice compression that results in zigzag or square structures, ED of molybdenum, schematic representation of electron diffraction tomography $(\mathrm{PDF})$

\section{AUTHOR INFORMATION}

\section{Corresponding Author}

*E-mail: d.vanmaekelbergh@uu.nl (D.V.).

\section{ORCID}

Joep L. Peters: 0000-0002-3415-648X

Carlo van Overbeek: 0000-0003-4244-4665

Sara Bals: 0000-0002-4249-8017

Daniel Vanmaekelbergh: 0000-0002-3535-8366

Notes

The authors declare no competing financial interest.

\section{ACKNOWLEDGMENTS}

The authors acknowledge funding from the European Commission (Grant EUSMI 731019). S.B. acknowledges funding from the European Research Council (Grant 335078 COLOURATOM). T.A. acknowledges a postdoctoral grant from the Research Foundation Flanders (FWO). The authors acknowledge financial support from the European Commission under the Horizon 2020 Programme by means of the Grant Agreement No. 731019 EUSMI.

\section{ABBREVIATIONS}

EG, ethylene glycol; NCs, nanocrystals; SAED, selected area electron diffraction; HAADF-STEM, high-angle annular darkfield scanning transmission electron microscopy.

\section{REFERENCES}

(1) Steckel, J. S.; Coe-Sullivan, S.; Bulović, V.; Bawendi, M. G. $1.3 \mu \mathrm{m}$ to $1.55 \mu \mathrm{m}$ Tunable Electroluminescence from PbSe Quantum Dots Embedded within an Organic Device. Adv. Mater. 2003, 15, $1862-1866$

(2) Ki Bae, W.; Kwak, J.; Lim, J.; Lee, D.; Ki Nam, M.; Char, K.; Lee, C.; Lee, S. Multicolored Light-Emitting Diodes Based on AllQuantum-Dot Multilayer Films Using Layer-by-Layer Assembly Method. Nano Lett. 2010, 10, 2368-2373.

(3) Wang, J. J.; Wang, Y. Q.; Cao, F. F.; Guo, Y. G.; Wan, L. J. Synthesis of Monodispersed Wurtzite Structure CuInSe2 Nanocrystals and Their Application in High-Performance Organic-Inorganic Hybrid Photodetectors. J. Am. Chem. Soc. 2010, 132, 12218-12221.

(4) Zhang, J.; Gao, J.; Church, C. P.; Miller, E. M.; Luther, J. M.; Klimov, V. I.; Beard, M. C. PbSe Quantum Dot Solar Cells with More than 6\% Efficiency Fabricated in Ambient Atmosphere. Nano Lett. 2014, 14, 6010-6015.

(5) Liu, Y.; Gibbs, M.; Perkins, C. L.; Tolentino, J.; Zarghami, M. H.; Bustamante, J.; Law, M. Robust, Functional Nanocrystal Solids by Infilling with Atomic Layer Deposition. Nano Lett. 2011, 11, 53495355.

(6) Zhitomirsky, D.; Furukawa, M.; Tang, J.; Stadler, P.; Hoogland, S.; Voznyy, O.; Liu, H.; Sargent, E. H. N-Type Colloidal-QuantumDot Solids for Photovoltaics. Adv. Mater. 2012, 24, 6181-6185.

(7) Nag, A.; Kovalenko, M. V.; Lee, J.; Liu, W.; Spokoyny, B.; Talapin, D. V. Metal-Free Inorganic Ligands for Colloidal Nanocrystals: S2-, HS-, Se2-, HSe-, Te2-, HTe-, TeS32-, OH-, and NH2- as Surface Ligands. J. Am. Chem. Soc. 2011, 133, 1061210620.

(8) Zhang, H.; Jang, J.; Liu, W.; Talapin, D. V. Colloidal Nanocrystals with Inorganic Halide, Pseudohalide, and Halometallate Ligands. ACS Nano 2014, 8, 7359-7369.

(9) Dolzhnikov, D. S.; Zhang, H.; Jang, J.; Son, J. S.; Panthani, M. G.; Shibata, T.; Chattopadhyay, S.; Talapin, D. V. CompositionMatched Molecular "Solders " for Semiconductors. Science 2015, 347, 425-428.

(10) Liu, Y.; Tolentino, J.; Gibbs, M.; Ihly, R.; Perkins, C. L.; Liu, Y.; Crawford, N.; Hemminger, J. C.; Law, M. PbSe Quantum Dot FieldEffect Transistors with Air-Stable Electron Mobilities above $7 \mathrm{Cm} 2 \mathrm{~V}$ 1 s-1. Nano Lett. 2013, 13, 1578-1587.

(11) Oh, S. J.; Berry, N. E.; Choi, J. H.; Gaulding, E. A.; Lin, H.; Paik, T.; Diroll, B. T.; Muramoto, S.; Murray, C. B.; Kagan, C. R. Designing High-Performance $\mathrm{PbS}$ and $\mathrm{PbSe}$ Nanocrystal Electronic Devices through Stepwise, Post-Synthesis, Colloidal Atomic Layer Deposition. Nano Lett. 2014, 14, 1559-1566.

(12) Evers, W. H.; Goris, B.; Bals, S.; Casavola, M.; De Graaf, J.; van Roij, R.; Dijkstra, M.; Vanmaekelbergh, D. Low-Dimensional Semiconductor Superlattices Formed by Geometric Control over Nanocrystal Attachment. Nano Lett. 2013, 13, 2317-2323.

(13) Baumgardner, W. J.; Whitham, K.; Hanrath, T. Confined-butConnected Quantum Solids via Controlled Ligand Displacement. Nano Lett. 2013, 13, 3225-3231. 
(14) Walravens, W.; De Roo, J.; Drijvers, E.; Ten Brinck, S.; Solano, E.; Dendooven, J.; Detavernier, C.; Infante, I.; Hens, Z. Chemically Triggered Formation of Two-Dimensional Epitaxial Quantum Dot Superlattices. ACS Nano 2016, 10, 6861-6870.

(15) Peters, J. L.; van den Bos, K. H. W.; Van Aert, S.; Goris, B.; Bals, S.; Vanmaekelbergh, D. Ligand-Induced Shape Transformation of PbSe Nanocrystals. Chem. Mater. 2017, 29, 4122-4128.

(16) Boneschanscher, M. P.; Evers, W. H.; Geuchies, J. J.; Altantzis, T.; Goris, B.; Rabouw, F. T.; van Rossum, S. A. P.; van der Zant, H. S. J.; Siebbeles, L. D. A.; Van Tendeloo, G.; Swart, I.; Hilhorst, J.; Petukhov, A. V.; Bals, S.; Vanmaekelbergh, D. Long-Range Orientation and Atomic Attachment of Nanocrystals in 2D Honeycomb Superlattices. Science 2014, 344, 1377-1380.

(17) Sarkar, S.; Acharya, S.; Chakraborty, A.; Pradhan, N. Zinc Blende 0D Quantum Dots to Wurtzite 1D Quantum Wires: The Oriented Attachment and Phase Change in $\mathrm{ZnSe}$ Nanostructures. J. Phys. Chem. Lett. 2013, 4, 3292-3297.

(18) O’Sullivan, C.; Gunning, R. D.; Sanyal, A.; Barrett, C. A.; Geaney, H.; Laffir, F. R.; Ahmed, S.; Ryan, K. M. Spontaneous Room Temperature Elongation of $\mathrm{CdS}$ and Ag2S Nanorods via Oriented Attachment. J. Am. Chem. Soc. 2009, 131, 12250-12257.

(19) Yu, M.; Draskovic, T. I.; Wu, Y. Understanding the Crystallization Mechanism of Delafossite $\mathrm{CuGaO} 2$ for Controlled Hydrothermal Synthesis of Nanoparticles and Nanoplates. Inorg. Chem. 2014, 53, 5845-5851.

(20) Whitham, K.; Yang, J.; Savitzky, B. H.; Kourkoutis, L. F.; Wise, F.; Hanrath, T. Charge Transport and Localization in Atomically Coherent Quantum Dot Solids. Nat. Mater. 2016, 15, 557-563.

(21) Delerue, C. From Semiconductor Nanocrystals to Artificial Solids with Dimensionality below Two. Phys. Chem. Chem. Phys. 2014, 16, 25734-25740.

(22) Delerue, C.; Vanmaekelbergh, D. Electronic Band Structure of Zinc Blende CdSe and Rock Salt PbSe Semiconductors with SiliceneType Honeycomb Geometry. 2D Mater. 2015, 2, 034008.

(23) Kalesaki, E.; Delerue, C.; Morais Smith, C.; Beugeling, W.; Allan, G.; Vanmaekelbergh, D. Dirac Cones, Topological Edge States, and Nontrivial Flat Bands in Two-Dimensional Semiconductors with a Honeycomb Nanogeometry. Phys. Rev. X 2014, 4, 011010.

(24) Kalesaki, E.; Evers, W. H.; Allan, G.; Vanmaekelbergh, D.; Delerue, C. Electronic Structure of Atomically Coherent Square Semiconductor Superlattices with Dimensionality below Two. Phys. Rev. B: Condens. Matter Mater. Phys. 2013, 88, 115431.

(25) Anderson, N. C.; Hendricks, M. P.; Choi, J. J.; Owen, J. S. Ligand Exchange and the Stoichiometry of Metal Chalcogenide Nanocrystals: Spectroscopic Observation of Facile Metal-Carboxylate Displacement and Binding. J. Am. Chem. Soc. 2013, 135, 1853618548 .

(26) Park, J.; Zheng, H.; Lee, W. C.; Geissler, P. L.; Rabani, E.; Alivisatos, A. P. Direct Observation of Nanoparticle Superlattice Formation by Using Liquid Cell Transmission Electron Microscopy. ACS Nano 2012, 6, 2078-2085.

(27) Lee, W. C.; Kim, B. H.; Choi, S.; Takeuchi, S.; Park, J. Liquid Cell Electron Microscopy of Nanoparticle Self-Assembly Driven by Solvent Drying. J. Phys. Chem. Lett. 2017, 8, 647-654.

(28) Rabani, E.; Reichman, D. R.; Geissler, P. L.; Brus, L. E. DryingMediated Self-Assembly of Nanoparticles. Nature 2003, 426, 271.

(29) Deegan, R. D.; Bakajin, O.; Dupont, T. F.; Huber, G.; Nagel, S. R.; Witten, T. A. Capillary Flow as the Cause of Ring Stains from Dried Liquid Drops. Nature 1997, 389, 827-829.

(30) Deegan, R. D. Contact Line Deposits in an Evaporation Drop.

Phys. Rev. 2000, 62, 756 DOI: 10.1103/PhysRevE.62.756.

(31) Hu, H.; Larson, R. G. Marangoni Effect Reverses Coffee-Ring Depositions. J. Phys. Chem. B 2006, 110, 7090-7094.

(32) Narayanan, S.; Wang, J.; Lin, X. M. Dynamical Self-Assembly of Nanocrystal Superlattices during Colloidal Droplet Evaporation by in Situ Small Angle x-Ray Scattering. Phys. Rev. Lett. 2004, 93, 1-4.

(33) Ohara, P. C.; Heath, J. R.; Gelbart, W. M. Self-Assembly of Submicrometer Rings of Particles from Solutions of Nanoparticles. Angew. Chem., Int. Ed. Engl. 1997, 36, 1078-1080.
(34) Yosef, G.; Rabani, E. Self-Assembly of Nanoparticles into Rings: A Lattice-Gas Model. J. Phys. Chem. B 2006, 110, 20965-20972.

(35) Witten, T. A.; Sander, L. M. Diffusion-Limited Aggregation, a Kinetic Critical Phenomenon. Phys. Rev. Lett. 1981, 47, 1400-1403.

(36) Udayabhaskararao, T.; Altantzis, T.; Houben, L.; CoronadoPuchau, M.; Langer, J.; Popovitz-Biro, R.; Liz-Marzán, L. M.; Vukovic, L.; Král, P.; Bals, S.; Klajn, R. Tunable Porous Nanoallotropes Prepared by Post-Assembly Etching of Binary Nanoparticle Superlattices. Science 2017, 358, 514-518.

(37) Tadjine, A.; Delerue, C. Colloidal Nanocrystals as LEGO® Bricks for Building Electronic Band Structure Models. Phys. Chem. Chem. Phys. 2018, 20, 8177-8184.

(38) Li, D.; Nielsen, M. H.; Lee, J. R. I.; Frandsen, C.; Banfield, J. F.; De Yoreo, J. J. Direction-Specific Interactions Control Crystal Growth by Oriented Attachment. Science 2012, 336, 1014-1018.

(39) Ijäs, M.; Ervasti, M.; Uppstu, A.; Liljeroth, P.; Van Der Lit, J.; Swart, I.; Harju, A. Electronic States in Finite Graphene Nanoribbons: Effect of Charging and Defects. Phys. Rev. B: Condens. Matter Mater. Phys. 2013, 88, 1-13.

(40) Bodnarchuk, M. I.; Shevchenko, E. V.; Talapin, D. V. Structural Defects in Periodic and Quasicrystalline Binary Nanocrystal Superlattices. J. Am. Chem. Soc. 2011, 133, 20837-20849.

(41) van Overbeek, C.; Peters, J. L.; van Rossum, S. A. P.; Smits, M.; van Huis, M. A.; Vanmaekelbergh, D. Interfacial Self-Assembly and Oriented Attachment in the Family of $\mathrm{PbX}(\mathrm{X}=\mathrm{S}$, Se, Te) Nanocrystals. J. Phys. Chem. C 2018, 122, 12464-12473.

(42) Geuchies, J. J.; van Overbeek, C.; Evers, W. H.; Goris, B.; de Backer, A.; Gantapara, A. P.; Rabouw, F. T.; Hilhorst, J.; Peters, J. L.; Konovalov, O.; Petukhov, A. V.; Dijkstra, M.; Siebbeles, L. D. A.; van Aert, S.; Bals, S.; Vanmaekelbergh, D. In-Situ Study of Consecutive Phase Transitions in the Formation of Atomically Coherent TwoDimensional Superlattices from Nanocrystals. Nat. Mater. 2016, 15, $1248-1254$.

(43) Goodfellow, B. W.; Patel, R. N.; Panthani, M. G.; Smilgies, D.M.; Korgel, B. A. Melting and Sintering of a Body-Centered Cubic $\mathrm{PbSe}$ Nanocrystal Superlattice Followed by Small Angle X-Ray Scattering. J. Phys. Chem. C 2011, 115, 6397-6404.

(44) Weidman, M. C.; Smilgies, D.-M.; Tisdale, W. A. Kinetics of the Self-Assembly of Nanocrystal Superlattices Measured by RealTime in Situ X-Ray Scattering. Nat. Mater. 2016, 15, 775-781.

(45) Bian, K.; Choi, J. J.; Kaushik, A.; Clancy, P.; Smilgies, D. M.; Hanrath, T. Shape-Anisotropy Driven Symmetry Transformations in Nanocrystal Superlattice Polymorphs. ACS Nano 2011, 5, 28152823.

(46) Penn, R. L. Imperfect Oriented Attachment: Dislocation Generation in Defect-Free Nanocrystals. Science 1998, 281, 969-971.

(47) Van Huis, M. A.; Kunneman, L. T.; Overgaag, K.; Xu, Q.; Pandraud, G.; Zandbergen, H. W.; Vanmaekelbergh, D. LowTemperature Nanocrystal Unification through Rotations and Relaxations Probed by in Situ Transmission Electron Microscopy. Nano Lett. 2008, 8, 3959-3963.

(48) Fang, J.; Ding, B.; Gleiter, H. Mesocrystals: Syntheses in Metals and Applications. Chem. Soc. Rev. 2011, 40, 5347-5360.

(49) Read, W. T.; Shockley, W. Dislocation Models of Crystal Grain Boundaries. Phys. Rev. 1950, 78, 275-289.

(50) Savitzky, B. H.; Hovden, R.; Whitham, K.; Yang, J.; Wise, F.; Hanrath, T.; Kourkoutis, L. F. Propagation of Structural Disorder in Epitaxially Connected Quantum Dot Solids from Atomic to Micron Scale. Nano Lett. 2016, 16, 5714-5718.

(51) van Aarle, W.; Palenstijn, W. J.; De Beenhouwer, J.; Altantzis, T.; Bals, S.; Batenburg, K. J.; Sijbers, J. Ultramicroscopy The ASTRA Toolbox: A Platform for Advanced Algorithm Development in Electron Tomography. Ultramicroscopy 2015, 157, 35-47. 